\title{
Molecular basis of the anti-cancer effects of genistein isoflavone in LNCaP prostate cancer cells
}

\section{${ }^{1}$ Merchant K, ${ }^{2}$ Kumi-Diaka J, ${ }^{3}$ Rathinavelu A, ${ }^{2}$ Esiobu N, ${ }^{4}$ Zoeller R, ${ }^{2}$ Hartmann J, ${ }^{2}$ Johnson M}

${ }^{1}$ College of Health Sciences, Barry University, 11300 NE $2^{\text {nd }}$ Ave Miami Shores FL 33161, USA

${ }^{2}$ Department of Biological Sciences, Florida Atlantic University, 3200 College Ave Davie FL 33314, USA

${ }^{3}$ Rumbaugh Goodwin Institute for Cancer Research, Nova Southeastern University, Plantation Fl, 33322, USA

${ }^{4}$ Department of Health \& Exercise Sci, Florida Atlantic University, 3200 College Ave Davie FL 33314, USA

Corresponding author: Kumi-Diaka J, PhD, Department of Biological Sciences, Florida Atlantic University, 3200 College Ave Davie FL 33314 USA

Submission date: 17 February 2011; Acceptance date: 31 March 2011; Publication date: 31 March 2011

\section{$\underline{\text { Abstract }}$}

Background: Prostate cancer is the most common form of non-skin cancer within the United States and the second leading cause of cancer deaths. Survival rates for the advanced disease remain relatively low, and conventional treatments may be accompanied by significant side effects. As a result, current research is aimed at alternative or adjuvant treatments that will target components of the signal transduction, cell-cycle and apoptosis pathways, to induce cell death with little or no toxic side effects to the patient. In this study, we investigated the effect of genistein isoflavone, a soy derivative, on expression levels of genes involved in these pathways. The mechanism of genistein-induced cell death was also investigated. The chemosensitivity of the $\mathrm{LNCaP}$ prostate cancer cells to genistein was investigated using ATP and MTS assays, and a caspase binding assay was used to determine apoptosis induction. Several molecular targets were determined using cDNA microarray and RT-PCR analysis.

Results: The overall data revealed that genistein induces cell death in a time- and dosedependent manner, and regulates expression levels of several genes involved in carcinogenesis and immunity. Several cell-cycle genes were down-regulated, including the mitotic kinesins, 
cyclins and cyclin-dependent kinases. Various members of the Bcl-2 family of apoptotic proteins were also affected. The DefB1 and the HLA membrane receptor genes involved in immunogenicity were also up-regulated.

Conclusion: The results indicate that genistein inhibits growth of the hormone-dependent prostate cancer cells, LNCaP, via apoptosis induction through regulation of some of the genes involved in carcinogenesis of many tumors, and immunogenicity. This study augments the potential phytotherapeutic and immunotherapeutic significance of genistein isoflavone.

Key words: Genistein isoflavone, prostate cancer, expression of genes, phytotherapeutic adjuvant, immunotherapy and chemotherapy

\section{Background}

Prostate cancer is the most common form of non-skin cancer and the second leading cause of cancer deaths in the United States [1]. The five year survival rate has increased from $69 \%$ to 99\% over the last 25 years for the local and regional disease, but has remained fairly low (approximately 34\%) for the advanced disease [1,2]. Known risk factors include age, race and family history, but other factors such as diet and lifestyle, environment and geographic location, may also play a role in the progression of this disease [3-6]. New diagnostic tools, improvement in diagnostic methods and increased testing for biologic markers, have contributed to early diagnosis and better treatment of the early-stage disease [7, 8].

Current research is aimed at finding new prognostic markers and targets for therapy. The apoptotic and cell cycle pathways provide many avenues for researchers and specific targets for cancer therapy. Many research efforts also focus on the effects of dietary phytochemicals. The goal of phytochemical research is to identify natural substances that will affect/modulate the growth regulatory (signal transduction-, cell-cycle-, growth factor-, and cell death) pathways in carcinogenesis. A major phytochemical that has been shown to have chemopreventive properties is genistein isoflavone. Genistein $\left(4^{\prime}, 5^{\prime}\right.$, 7-trihydroxyiso-flavone) is a major isoflavone constituent of soy that is found naturally as the glycoside genistin. The latter is hydrolyzed to genistein by natural microflora in the intestine.

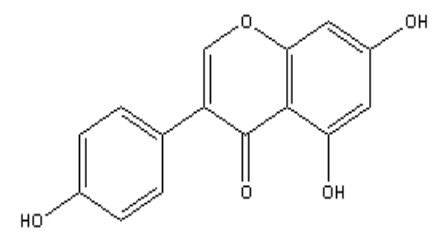

The molecular structure of genistein

Genistein is structurally similar to estrogen and therefore possesses the ability to bind to estrogenic receptors and regulate cell proliferation [9 -11]. Studies have shown that genistein also exhibits several pharmacological effects, including the inhibition of: tyrosine kinases, DNA 
topoisomerases, microsomal lipid peroxidation, and angiogenesis [10-12]. Genistein has been shown to act as an antioxidant and a cell cycle inhibitor, by arresting the cell cycle at the G2/M phase $[11,13]$. Physiological levels of genistein vary depending on diet consumption, but levels ranging from 0.2 to $9.0 \mu \mathrm{mol}$ have been found $[14,15]$. In our study, we focused on using genistein levels that might be achieved through use of a supplement or as adjuvant to standard therapeutic regimen/treatments such as chemotherapy and/or radiation therapy.

\section{Materials and Methods}

\section{Cell culture}

LNCaP cells were cultured in RPMI-1640 growth medium supplemented with $10 \%$ FBS, $1 \%$ penicillin/streptomycin and L-glutamine. Cells were incubated for $24-48 \mathrm{hrs}$ at $37^{\circ} \mathrm{C}$ and $5 \%$ $\mathrm{CO}_{2}$ to achieve $60-80 \%$ confluence. After incubation, the medium was removed and either replaced with medium alone, as a negative control, or medium containing various concentrations of genistein $(25,50,75,100 \mu \mathrm{M})$, and incubated for 24-48 hrs. Genistein (4', 5', 7trihydroisoflavone (Indofine Chem. Co., Inc. Summerville, NJ, USA) was dissolved in dimethylsulfoxide (DMSO) as a $100 \mu \mathrm{M}$ stock solution and diluted in RPMI-1640 culture medium at a final DMSO concentration of $0.25 \%(\mathrm{vol} / \mathrm{vol})$; genistein $(\mathrm{Gn})$ concentrations of 0 , $25,50,75,100 \mu \mathrm{M}\left(\mathrm{Gn}_{0-100}\right)$ were prepared and stored at $-35^{\circ} \mathrm{C}$. In previous preliminary studies, the $\mathrm{IC}_{50}$ for genistein was observed at 25-30 $\square \mathrm{M}$ in PC3 and LNCaP cells. DMSO alone was found to be non-toxic to these cells at up to $0.5 \%$.

\section{ATP Assay}

The cytotoxicity of genistein on LNCaP cells was determined using the ATP assay. The assay utilizes the D-luciferin substrate which, when oxidized in an ATP-dependent process catalyzed by firefly luciferase, generates chemiluminiscence at $560 \mathrm{~nm}$. The assay is optimized for fast determination of low levels of ATP. Concentrations down to 0.1 pmol can be accurately determined using the luminescent signal of the luciferase reaction.

For determination, cells were plated at a density of $5 \times 10^{4}$ cells per well in a 96-well plate and incubated for $24-36 \mathrm{hr}$ to achieve confluence. After incubation, the cells were treated with varying concentrations of genistein, or $1 \%$ Triton-X as a positive control. The cells were then incubated for $24 \mathrm{hrs}$ under standard conditions. After incubation, $50 \mu \mathrm{l}$ of lysis solution was immediately added to each well. After $5 \mathrm{~min}, 50 \mu \mathrm{l}$ of solution was transferred from each well of the 96-well plate to its corresponding well in a white plate. Fifty $\mu$ l of luciferin/luciferase solution was then added to each well. Luminescence was determined after 5 min using a luminometer. ATP concentration was determined by comparison of luminescent values against a standard curve and \% inhibition was calculated.

\section{MTS Assay}

The MTS assay was also performed to determine the cytotoxicity of genistein towards LNCaP cells. This assay utilizes MTS (3-(4, 5-dimethylthiazol-2-yl)-5-(3-carboxymethoxyphenyl)-2-(4- 
sulfophenyl)-2H-tetrazolium, to determine cell viability. In the presence of viable cells, the substrate is reduced to a colored formazan product. In active mitochondria, the tetrazolium ring is cleaved in the presence of dehydrogenase enzymes, producing a product that is soluble in tissue culture medium. The color intensity is directly proportional to the number of viable cells; therefore, relative cell numbers can be determined based on optical absorbance from each well of the microtiter plate.

Briefly, cells were plated at a density of $5 \times 10^{4}$ cells per well in a 96-well plate and incubated for $24 \mathrm{hrs}$ to achieve confluence. After incubation, the cells were treated with varying concentrations of genistein, or $1 \%$ Triton-X as a positive control. After 48 hrs incubation, $10 \mu \mathrm{l}$ of MTS reagent was added to each well of the 96-well plate. The plates were then incubated at $37^{\circ} \mathrm{C}$ and $5 \% \mathrm{CO}_{2}$ for $4 \mathrm{hrs}$ and the absorbance was read at $490 \mathrm{~nm}$ using a plate reader. Percent inhibition was then calculated.

\section{Apoptosis Induction}

Apoptosis is characterized by morphological changes within the cells, such as, fragmentation of nuclear chromatin and cell shrinkage. These changes can be detected by exposing the cells to certain dyes which, when taken up by the cells, cause emission of different fluorescent spectra that indicate the level of cell death. Apoptosis induction was determined using the Vybrant ${ }^{\circledR}$ apoptosis assay kit. This assay utilizes two dyes, Propidium Iodide (PI) and YO-PRO®-1. The green fluorescent dye YO-PRO ${ }^{\circledR}-1$ can enter early apoptotic cells, whereas the red fluorescent dye PI will only enter dead cells. Therefore, use of both dyes provides a sensitive indication of apoptosis. After staining cells with both dyes, live cells show little or no fluorescence, early apoptotic cells will show green fluorescence and dead cells (membrane compromised cells) will show both red and green fluorescence. These can be detected by fluorescence microscopy or flow cytometry.

Cells were harvested and plated in 6-well plates and incubated for $24 \mathrm{hrs}$ to achieve confluence. After incubation, cells were treated with varying concentrations of genistein, or topotecan $(50 \mu \mathrm{M})$ as a positive inducer of apoptosis. The cells were then incubated for $48 \mathrm{hrs}$. After incubation, cells were harvested and washed with cold phosphate buffered saline (PBS), and cell density was adjusted to $1 \times 10^{6}$ cells/ml in PBS. $1 \mu 1$ of YO-PROß-1 stock solution (Component A) was added followed by $1 \mu \mathrm{l}$ of PI stock solution (Component B), to each $1 \mathrm{ml}$ of cell suspension. The cells were incubated on ice for 20-30 min, then analyzed on the Accuri C6 flow cytometer ${ }^{\circledR}$ using $488 \mathrm{~nm}$ excitation with green fluorescence emission for YO-PRO ${ }^{\circledR}-1$ and red fluorescence emission for PI. Data was then analyzed using the CFlow Plus ${ }^{\circledR}$ software.

\section{Caspase Protease Analysis}

The involvement of caspase-3 in the molecular pathway of genistein-induced apoptosis was determined by using a caspase-3 colorimetric activity assay. The assay involves the caspase mediated cleavage of a labeled peptide substrate acetyl-Asp-Glu-Val-Asp p-nitroanilide (Ac- 
DEVD- $p$ NA). The $p$-nitroaniline ( $p$ NA) product can be quantified using a spectrophotometer or plate reader at $405 \mathrm{~nm}$. This gives a direct indication of caspase- 3 activity.

For caspase-3 determination, the cells were treated with varying concentrations of genistein or topotecan $(50 \mu \mathrm{M})$ as a positive inducer of apoptosis. The cells were counted and collected by centrifugation at $1500 \mathrm{rpm}$ for $5 \mathrm{~min} .2 \times 10^{6}$ cells were then resuspended in $200 \mu 1$ of lysis buffer and incubated on ice for $20 \mathrm{~min}$. After incubation, the solution was centrifuged for $5 \mathrm{~min}$ at $10,000 \mathrm{x} \mathrm{g}$ and the supernatant was collected. The BCA protein assay kit (Pierce) was used to determine the protein concentration of the samples. $30 \mu \mathrm{g}$ of protein lysate for each sample was then assayed for caspase activity. Fold-increase in caspase- 3 activity was then calculated by comparing the absorbance values of the treated samples to that of the control sample.

\section{cDNA Microarray Analysis}

After treatment of the LNCaP cells with $100 \mu \mathrm{M}$ genistein, the RNA was extracted using the RNeasy mini kit (Qiagen) according to the manufacturer's protocol. RNA quality was determined by separation on a $1 \%$ agarose gel and confirmed by spectrophotometry. Good quality RNA was obtained by observing the $18 \mathrm{~S}$ and $26 \mathrm{~S}$ bands on the agarose gel, and by using absorbance values which gave an $\mathrm{A}_{260 / 280}$ ratio between 1.8 and 2. RNA samples were then submitted to the Genomics Core of the Scripps Research Institute (Jupiter, FL) for microarray analysis.

Briefly, RNA samples were quantified using the NanoDrop ND-1000. Double-stranded cDNA was prepared from $1 \mu \mathrm{g}$ of total RNA using the Affymetrix cDNA synthesis kit, and in vitro transcribed using an IVT labeling kit (Affymetrix), with the cRNA product purified using a GeneChip Sample Cleanup Module (Affymetrix). $20 \mu \mathrm{g}$ biotin-labeled cRNA was fragmented and hybridized to Affymetrix Human Gene chip U133 plus 2.0 array overnight in the Affy 640 hybridization oven, with a speed of $60 \mathrm{rpm}$ for $16 \mathrm{hr}$. Microarrays were washed and stained using the Affymetrix Fluidics Station FS400.

GeneChip arrays were scanned using a GeneChip Scanner 3000 (Affymetrix). The probe set intensities were quantified using the GeneChip Operating Software (GCOS) and analyzed with GCRMA normalization using Array Assist Software (Stratagene, La Jolla, CA). All hybridized chips met standard quality control criteria, and mean fluorescence values of each array were scaled to a mean intensity of 500. Gene expression of genistein treated cells was compared with control (untreated) cells to determine changes in expression of target genes. Functional cluster analysis was done using the Database for Annotation, Visualization and Integrated Discovery (DAVID) ${ }^{(16)}$. Results of the microarray analysis were verified by RT-PCR.

\section{RT-PCR Analysis}

The RNA extracted from the cell samples was subjected to RT-PCR analysis using the Access RT-PCR System (Promega). Selected genes were reverse transcribed to verify the results of the 
array gene expression changes. The Access RT-PCR System is designed for convenient and sensitive detection and analysis of RNA by one-step RT-PCR. cDNA synthesis and PCR are performed in a single tube using gene specific primers $(4 \mu \mathrm{M})$ and $1 \mu \mathrm{g}$ of total RNA and amplified for 35 cycles. The specific primers used were bax, bcl-x, vegf, mdm-2 and beta-actin as an internal loading control. The amplified products were separated on a 1\% agarose gel stained with ethidium bromide. The results of this experiment were compared to that of the microarray analysis to determine if similar changes in gene expression were observed in target genes.

\section{Statistical Analysis}

Experiments were performed in duplicates to confirm similar results. Significance of the differences in mean values was determined using the Student's t-test and considering $\mathrm{P}<0.05$ to be statistically significant.

\section{Results}

\section{Growth inhibition}

The MTS and ATP assays were done to determine the cytotoxicity of genistein on LNCaP cells. The data obtained shows that genistein is able to induce cell death, which is consistent with previous studies. The ATP assay showed a steady increase in growth inhibition as genistein concentration increased [Figure 1].

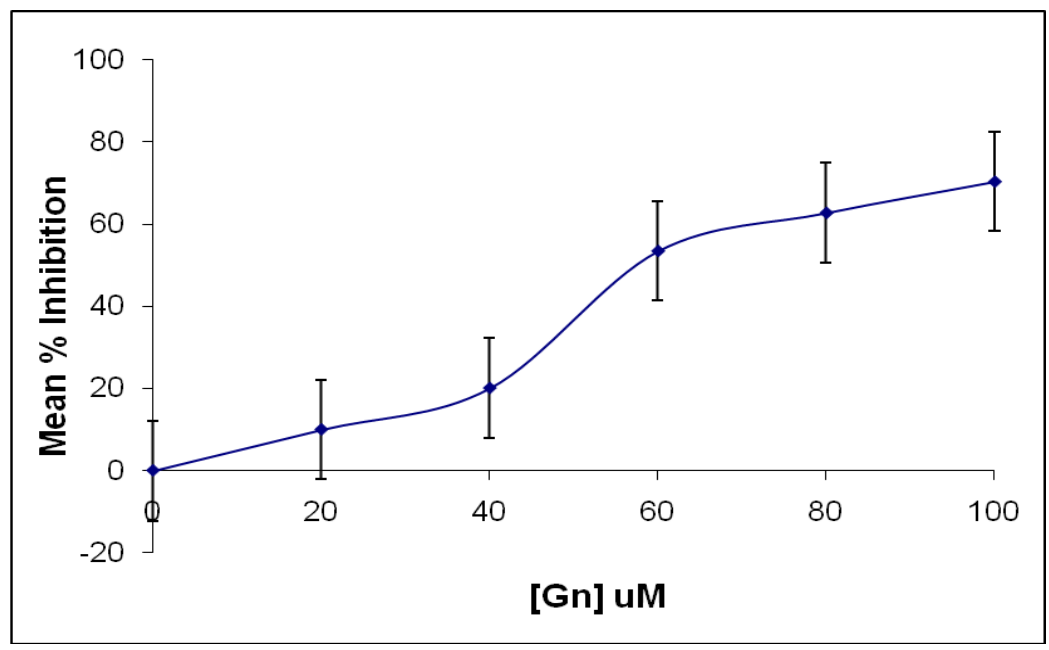

Figure 1. Determination of cytotoxic effect of genistein on growth inhibition of LNCaP cells, using the ATP assay. Cells were treated with varying concentrations of genistein and \% inhibition was determined using the ATP assay. Data are representative of two independent experiments and are expressed as the mean \pm SEM. Bar $=$ SEM

Being a more sensitive assay, we were able to see a significant difference after just 24 hrs of treatment. There was a significant increase in growth inhibition of the cells from 0 to 60 
$\mu \mathrm{M}(\mathrm{p}<0.05)$. Above $60 \mu \mathrm{M}$, there was a further increase in inhibition but this was not significant $(\mathrm{p}>0.05)$.

The MTS assay also showed a steady increase in inhibition [Figure 2]. At 24 hrs there was no significant increase from 25 to $50 \mu \mathrm{M}$ ( $\mathrm{p}>0.05$ ). Above $50 \mu \mathrm{M}$, there was a significant increase in growth inhibition in the cells $(\mathrm{p}<0.05)$. At $48 \mathrm{hrs}$, there was a significant increase in growth inhibition from 0 to $50 \mu \mathrm{M}$ ( $\mathrm{p}<0.05$ ), but no significant increase was observed beyond that concentration ( $p>0.05$ ). At $25 \mu \mathrm{M}$, there was no difference between the effect seen at 24 and $48 \mathrm{hrs}$, but at higher concentrations, the difference in growth inhibition observed was significantly higher at $48 \mathrm{hrs}(\mathrm{p}<0.05)$. This provides further evidence that the effects of genistein are both time- and dose-dependent. The $\mathrm{EC}_{50}$ of genistein in LNCaP cells was determined, from the data obtained, to be about 50-60 $\mu \mathrm{M}$.

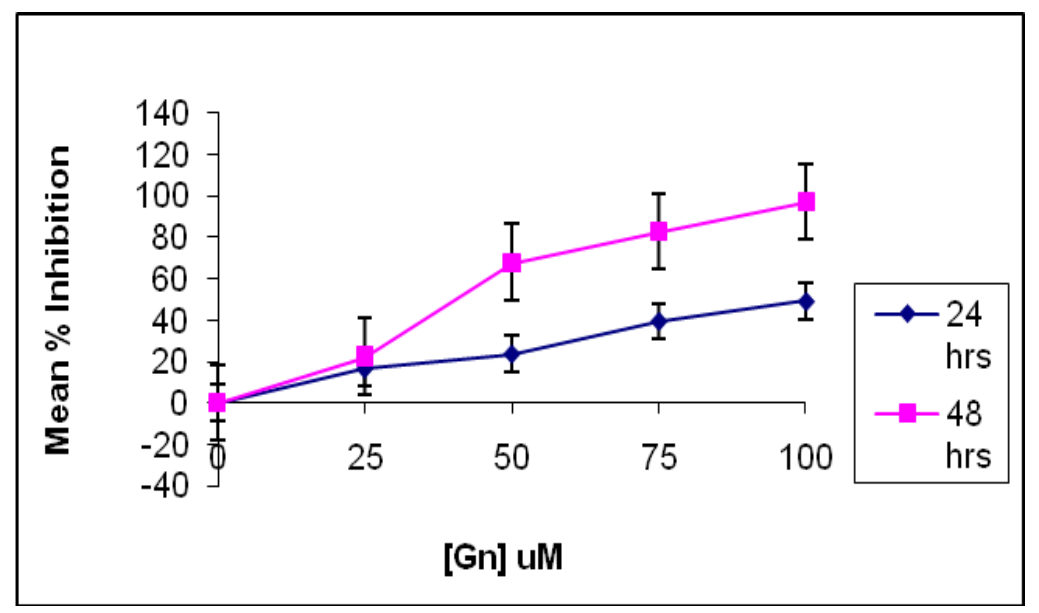

Figure 2. Determination of the cytotoxic effect of genistein on growth inhibition of LNCaP cells, using the MTS assay. Cells were treated with varying concentrations of genistein and \% inhibition was determined using the MTS assay. Data are representative of two independent experiments and are expressed as the mean \pm SEM. Bar $=$ SEM

\section{Apoptosis Induction and Caspase-3 induction}

Apoptosis induction in genistein treated cells was investigated and the results show that genistein has a dose-dependent effect on LNCaP cells. Flow cytometer analysis of cells stained with the fluorescent dyes YO-PRO ${ }^{\circledR}-1$ and PI showed an increased level of apoptosis with increasing genistein concentration [Figure 3]. This figure shows a comparison of the population of live and apoptotic cells that were observed after treatment with genistein. The data show that genistein induces apoptosis and inhibits growth of cells, and this response is dose-dependent, as seen with the ATP and MTS assays. Data obtained from the caspase-3 assay indicate that caspase-3 induction increases in a time- and dose-dependent manner. Experiments were performed in the presence and absence of a caspase- 3 inhibitor and the data show that there was a significant difference in caspase-3 activity between the two treatment groups. In the presence of the caspase-3 inhibitor, expression was the same after $24 \mathrm{hrs}$, even at high concentrations of 
genistein (Figure 4). However, in the absence of any inhibition there was a 1.5-fold increase at $50 \mu \mathrm{M}$ after just $24 \mathrm{hrs}$, and almost a 5-fold increase at the same concentration, after $48 \mathrm{hrs}$ (data not shown). These results correlate with the data obtained in the apoptosis assay, and indicate that genistein-induced cell death involves the activation of the caspase pathway.

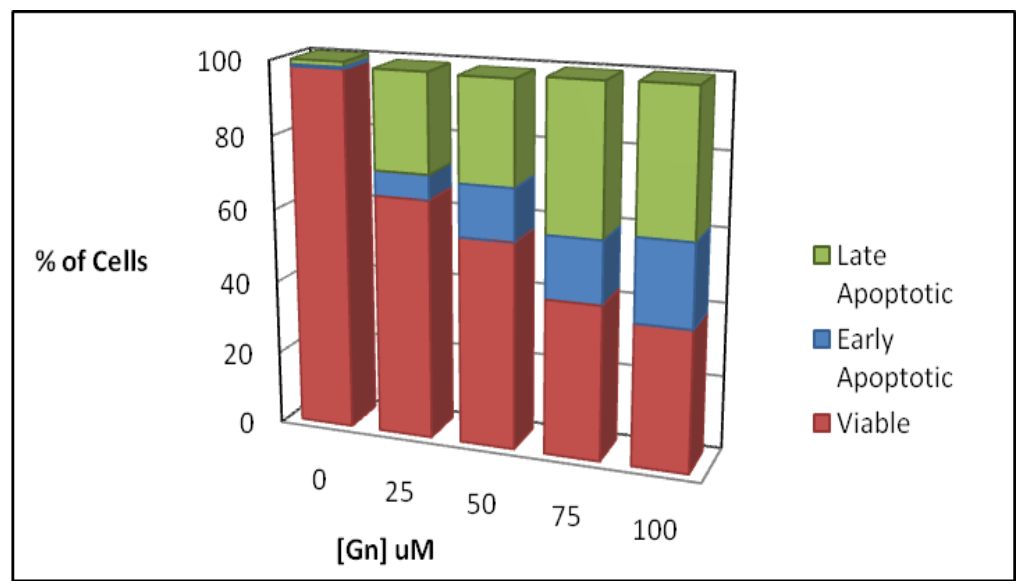

Figure 3. Bar chart showing distribution of viable and apoptotic cells with respect to concentration of genistein. Cells were treated with varying concentrations of genistein and analyzed by flow cytometry, using YO-PRO ${ }^{\circledR}-1$ and PI stains to detect apoptosis induction. Data is representative of two independent experiments.

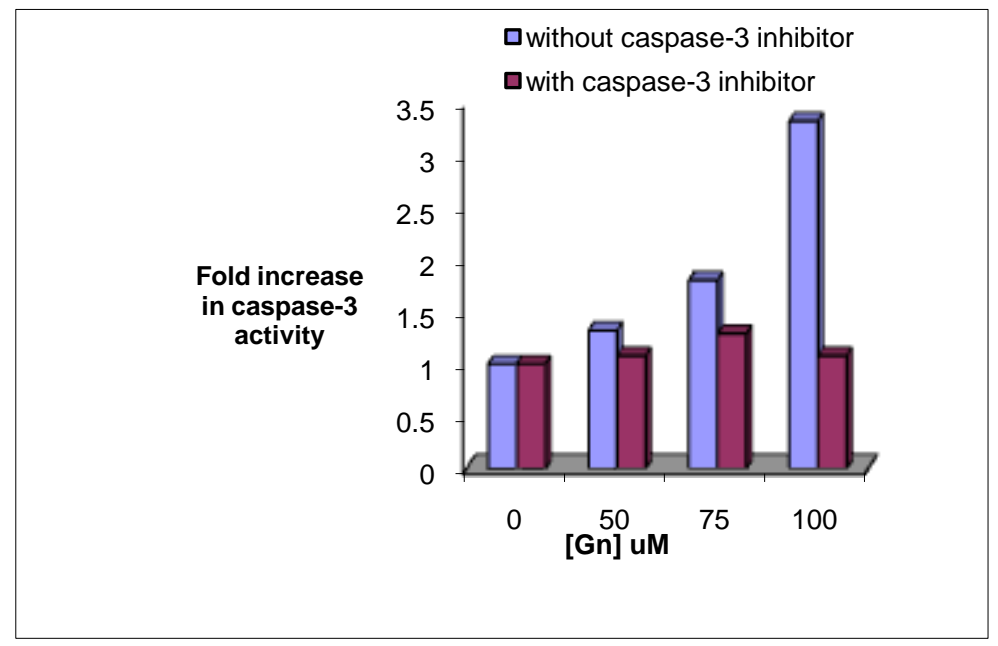

Figure 4. Effect of genistein treatment on caspase-3 activity in LNCaP cells after 24 hrs. Cells were treated and changes in caspase- 3 activity were determined. Data is representative of two independent experiments.

\section{Microarray Analysis}

The effect of genistein on gene expression in LNCaP cells was investigated using microarray analysis. The Affymetrix human gene chip U133 plus 2.0 array was used to compare expression 
of genes in genistein treated and untreated LNCaP cells. A 2-fold difference in expression, compared to the control, was considered significant. A functional annotation cluster analysis of all these genes was performed, and changes in specific pathways were investigated based on KEGG annotations [17]. A number of pathways were found to be affected by genistein treatment. The cell cycle pathway was the most affected, with 51 genes being down-regulated (data not shown). The p53 signaling pathway was also affected with 14 genes being up-regulated and 14 being down-regulated.

The main cell cycle genes affected included the kinesins, cyclins and cyclin-dependent kinases that are involved in regulation of the cell cycle (Table 1).

Table 1. Cell cycle genes showing genistein-induced differential expression

\begin{tabular}{|l|}
\hline Down regulated genes \\
\hline Cyclins B1, B2, E1, E2, F \\
Cdks 2, 3, 4, 5 \\
Tp53 (Li Fraumeni syndrome) \\
TGFB2 \\
Tfdp1 \\
\hline Up regulated genes \\
\hline Mdm2 \\
TP53AP1 \\
TP53INP2 \\
CDKN1A \\
CDKN2B \\
\hline
\end{tabular}

Other genes that were down-regulated include TGFB2, and TP53 Li Fraumeni (the mutated form of the p53 gene), which are known to be involved in tumor progression. Upregulated genes include p53 related proteins, mdm-2 and cdk inhibitors $1 \mathrm{a}$ and $2 \mathrm{~b}$ (Table 1). A number of apoptosis related genes were affected as well (Table 2), including members of the Bcl-2 family.

Table 2. Apoptosis-related genes showing genistein-induced differential expression

\begin{tabular}{|l|}
\hline Down regulated genes \\
\hline Bcl-2, Bcl-x, BCLAF-1, \\
BCL11A \\
NFKB1 \\
FAIM2 \\
MAP2K5 \\
\hline Up regulated genes \\
\hline Fas \\
TNFSF9 \\
BIRC3 \\
BAM (BIM/Bcl2L11) \\
\hline
\end{tabular}

Bcl-2 and Bcl-x, which are anti-apoptotic members, were found to be down-regulated following genistein treatment, and Bam, which is an apoptosis inducer, was found to upregulated. Genistein was found to have no effect on Bax, maspin, and VEGF expression levels. 
Other genes that were affected include members of the TNF superfamily, which are involved in the apoptotic pathway. In addition, the DefB1 and the HLA membrane receptor genes involved in immunogenicity were also up-regulated.

Down-regulated genes showing a 20-fold or higher expression were further analyzed using the DAVID annotation tool and a functional annotation cluster was performed. The results revealed three gene clusters containing genes with similar functions (Table 3).

Table 3. Functional cluster analysis of genes showing decreased expression of 20 -fold or less and increased expression of 10-fold or more

\begin{tabular}{|l|}
\hline Down regulated genes \\
\hline Cluster 1 \\
KIF2C, KIF4A, KIF14, KIF15, KIF20, KIF23 \\
BUB1, PLK1, PLK4, CENPO, CENPF \\
ESPL1, DLG7, TTK, NEK2, SPC25 \\
Cluster 2 \\
MCM10, NEIL3, ST18 \\
Cluster 3 \\
E2F2, E2F8 \\
\hline Up regulated genes \\
\hline NEU1 (sialidase1), CD68, Insig1, GPNMB \\
\hline
\end{tabular}

Group 1 had 24 genes including the kinesins and a number of other cell-cycle related genes. Groups 2 and 3 both had 3 genes; the other 38 genes were omitted from the cluster by the program. In group 1, the kinesins were significantly affected showing fold-changes ranging from 22 to 87. The second group included the MCM10 gene, which interacts with the origin of recognition complex (ORC) and two other regulatory proteins, CDC6 and CDT1, in formation of the pre-replication complex. The third group contained genes that code for the transcription factors, E2F2 and E2F8, which are involved in transcription of cell cycle related genes.

Up-regulated genes having a 10-fold or higher differential change in expression levels were also analyzed further, and clustered according to function. Only one functionally similar group of genes was found which included CD68, GPNMB and NEU1, which seem to play a role in immune cell recognition of the cancer cells. BrunoL6 gene was not included in the cluster, but this gene was the most up-regulated gene, showing a fold-change of 116.0.

\section{RT-PCR Analysis}

A few genes were selected and subjected to RT-PCR analysis to confirm the results of the microarray. The selected genes include Bax, Bcl-x, Mdm-2, VEGF and $\beta$-actin (which was used as a loading control). The results indicate that Mdm-2 showed an increase in expression when cells were treated with genistein; which correlates with the microarray results. VEGF and Bax expressions showed no significant changes after genistein treatment; which also correlates with the microarray results. Bcl-x showed a slight decrease in expression level as revealed by both 
PCR and microarray analysis. These findings indicate that the PCR results are consistent with the microarray data obtained, and confirm the effects of genistein on gene expression within the prostate cancer cells.

\section{Discussion}

\section{Growth Inhibition of LNCaP Cells}

Genistein has been shown to induce cell death in cancer cells through apoptosis; and previous studies have indicated that this is a time- and dose-dependent effect [18-20]. The results of the ATP and MTS proliferation assays are consistent with these studies and indicate the chemosensitivity of LNCaP prostate cancer cells towards genistein. Based on our results, the $\mathrm{EC}_{50}$ of genistein was found to be between 50 and $60 \mu \mathrm{M}$, which is also consistent with the results of previous studies [20]. Therefore, our study used a concentration range close to the $\mathrm{EC}_{50}$ value that was found in our preliminary studies.

These concentrations are above the physiological values observed in persons that have a high soy diet. For our studies, we wanted to look at the efficacy of concentrations that may be present in persons taking a genistein supplement or using it as an adjuvant drug.

\section{Apoptosis and caspase-3 induction}

The results of the apoptosis and caspase-3 assays revealed that genistein can induce apoptosis and increase caspase-3 activity within the cells, as shown previously [21, 22]. Genistein's effect on apoptosis in the LNCaP cells was found to be dose-dependent, as observed from the results of the flow cytometry experiment. Cells in early apoptosis were detected as low as $25 \mu \mathrm{M}$ of genistein treatment and this percentage increased with the concentration of genistein.

Caspase- 3 activity was also increased after exposure to genistein. Caspase- 3 is an effector caspase and plays a central role in apoptotic cell death, so detection of this caspase indicates activity of other up-stream caspases. Once activated, caspase-3 has been shown to initiate fragmentation of DNA which ultimately leads to cell death [22].

The microarray analysis revealed that there was no change in expression levels of caspases. Since caspases are synthesized and found in the cell as inactive proteins, this could indicate that genistein increases activity of the protein but has no effect on gene expression levels.

\section{Gene Expression Profiles}

The microarray results revealed that a number of genes were affected/regulated by genistein. Several major pathways were shown to be affected including: the cell-cycle pathway, p53 signaling pathway, and apoptosis pathways. From previous studies, we know that genistein affects gene expression, even at low concentrations. One study found that at physiologically achievable concentrations of genistein $(<10 \mu \mathrm{M})$, some pathways were affected, but other pathways, such as the cell-cycle, DNA damage and stress response pathways, needed higher concentrations to elicit a significant response; in conformity with the present results [23]. 
Another study showed that at very high genistein concentrations $(100 \mu \mathrm{M})$, not more than four genes were down-regulated, and only seven genes were up-regulated by more than a 2-fold change in expression [24]. This suggests that finding the $\mathrm{EC}_{50}$, and using concentrations close to that value will have more of an effect on cancer cells, while still being less toxic to normal cells, as observed in this study.

\section{Down-regulation of Genes by Genistein}

The majority of down-regulated genes were found to be involved in the cell-cycle, suggesting that the major mechanism of treatment-induced cell death involves regulation of genes that control the cell-cycle. If cell-cycle genes are down-regulated, this will halt progression of the cell-cycle, leading to apoptosis within the cell. In the present study, the main group of cell cycle genes affected was comprised of kinesins. Kinesins are a large super-family of motor proteins that are involved in a wide range of cellular processes such as mitosis, meiosis and cargo transport. Our data showed that mostly the mitotic kinesins were affected. This further indicates the mechanistic specificity of genistein treatment.

Our data revealed that cyclin A2 was the most down-regulated gene (25.9- fold change) from this family. The G2/M checkpoint is controlled by interaction of cyclin $\mathrm{A} / \mathrm{cdk} 2$, and silencing of this gene was found to delay entry into mitosis [25], while over-expression was associated with poor prognosis in breast cancer patients [26]. Cyclin B1 and B2 were also downregulated, which, according to a study investigating the correlation between cyclin $\mathrm{B}$ levels and cell-cycle progression, co-depletion of both cyclins can result in mitotic arrest within the cells [27].

The anti-apoptotic proteins Bcl-2 and Bcl-x were down-regulated and Bim was upregulated by genistein. A decrease in Bcl-x expression was observed using both microarray and PCR analysis. Cell survival depends on a proper balance of these proteins; therefore, effective agents can be described as those that shift the balance in favor of apoptosis [28]. As seen from our results, genistein-induced cell death is aided by an increase in expression of pro-apoptotic member, Bim and a decrease in expression of anti-apoptotic members, Bcl-x and Bcl-2. Gene expression of Bax showed no significant change following genistein treatment (which was confirmed by PCR analysis), although protein levels were increased (results not shown). This suggests possible involvement of genistein in post-translational activation of Bax.

MMPs and VEGF play a major role in cancer metastasis and previous studies correlated genistein treatment with decreased protein activity [29, 30]. Presently however, the microarray and PCR analysis revealed no significant changes in gene expression levels following the treatment, which may indicate involvement of genistein in post-translational activation of these genes.

\section{Up-regulation of Genes by Genistein}

Bruno-L6 was the most up-regulated gene, showing a fold-change of 116.0. This gene plays a role in mRNA processing and alternative splicing, and belongs to a family of proteins that 
regulate mRNA stability and translation [31]. A study of the Xenopus homolog of this gene revealed that it mediates sequence specific deadenylation and repression of Eg5 (Kinesin5) [32], indicating that up-regulation of this gene by genistein may play a role in the down-regulation of kinesins. TP53INP1, TP53INP2 and TP53AP1 were also found to be up-regulated by genistein. TP53INP1 regulates p53 activity and is expressed during cellular stress to induce apoptosis [33]. TP53AP1 is a p53 target protein that also functions in response to cellular damage [34].

In our study, Mdm-2, which inhibits p53 activity, was also up-regulated by genistein (3.7- fold changes). This expression change was confirmed using PCR analysis. Increased expression of Mdm-2 was unexpected and may explain why an increase in the expression levels of these p53-associated proteins was not observed. Without increased Mdm-2 expression, a greater effect on apoptotic induction may have been apparent.

\section{Conclusion}

Our study provides evidence that genistein affects expression of genes that are involved in carcinogenesis. These results imply that genistein as a phytotherapeutic adjuvant may augment standard immunotherapy and chemotherapy, help to reduce toxic side-effects, and increase the patient's response to therapy. A number of other molecular targets were also identified, and further research will enable us to elucidate the significance of these genes and further mechanisms of genistein-induced apoptosis in prostate cancer cells.

\section{Authors' contribution}

KM and JD designed the study; KM and MJ collected and analyzed the data; all authors contributed to interpretation of data and reviewed the manuscript. None of the authors had a conflict of interest. None of the authors has conflicting interest in this program.

\section{Acknowledgements}

The authors acknowledge the material and partial financial support of Florida Atlantic University. The collaboration and material support of Rambough-Goodwin Cancer Research Institute of Nova Southeastern University is highly acknowledged. We also recognize the technical and research assistance of our laboratory staff.

\section{References}

1. Jemal A, Siegel R, Ward E et al. (2008) Cancer Statistics 2008. CA Cancer J Clin 58, 71-96.

2. Stanford JL, Stephenson RA, Coyle LM et al. (1998) Prostate Cancer Trends 1973-1995, SEER Program, National Cancer Institute. NIH Pub. No. 99-4543. Bethesda, MD.

3. Mettlin C. (1997). Recent developments in the epidemiology of prostate cancer. Eur J Cancer 33(3):340-347. 
4. Marks LS, Kojima M, Demarzo A et al. (2004) Prostate Cancer in Native Japanese and Japanese American Men: Effects of Dietary Differences on Prostatic Tissue. Urology 64, 765-771.

5. Colli JL, Colli A. (2005) International comparisons of prostate cancer mortality rates with dietary practices and sunlight levels. Urol Oncol 23, 390-398.

6. Klassen AC, Platz EA. (2006) What Can Geography Tell Us About Prostate Cancer? Am J Prev Med 30, S7-S15?

7. Gao X, Porter AT, Grignon DJ et al. (1997) Diagnostic and Prognostic Markers for Human Prostate Cancer. Prostate 31, 264-281.

8. Baade PD, Coory MD, Aitken JF. (2004) International trends in prostate-cancer mortality: the decrease is continuing and spreading. Cancer Causes Control 15, 237-241.

9. Hasler CM, Blumberg JB. (1999) Symposium on Phytochemicals: Biochemistry and Physiology. American Society for Nutritional Sciences. 129, 756S-757S.

10. Dixon RA, Ferreira D. (2002) Genistein Phytochemistry 60, 205-211.

11. Sarkar FH, Yiwei L. (2002) Mechanisms of cancer chemoprevention by soy isoflavone genistein. Cancer Metastasis Rev 21, 265-280.

12. Dalu A, Haskell JF, Coward L et al. (1998) Genistein, a component of soy, inhibits the expression of EGF and ErbB2/Neu receptors in the rat dorsolateral prostate. Prostate 37, 3643.

13. Chen A, Donovan SM. (2004) Genistein at a Concentration Present in Soy Infant Formula Inhibits Caco-2BBe Cell Proliferation by Causing G2/M Cell Cycle Arrest. J Nutr 134, 13038.

14. Xu X, Harris K, Wang $\mathrm{H}$ et al. (1995) Bioavailability of soybean isoflavones depends upon gut microflora in women. $J$ Nutr 125, 2307-2315.

15. Adlercreutz H, Markkanen H, Watanabe S. (1993) Plasma concentrations of phyto-estrogens in Japanese men. Lancet 342, 1209-10.

16. Glynn D, Sherman BT, Hosack DA et al. (2003) DAVID: Database for Annotation, Visualization, and Integrated Discovery. Genome Biol 4, P3.

17. Kanehisa M, Goto S, Kawashima S et al. (2002) The KEGG databases at Genome Net. Nucleic Acids Res 30, 42-46.

18. Kumi-Diaka J, Saddler-Shawnette S, Aller A et al. (2004) Potential mechanism of phytochemical-induced apoptosis in human prostate adenocarcinoma cells: Therapeutic synergy of genistein and $\beta$-lapachone combination treatment. Cancer Cell Int 4:5.

19. Merchant K. (2005) Anticancer Activity of Two Dietary Phytochemicals: Genistein Isoflavone and $\beta$-Lapachone. Master's thesis, Florida Atlantic University.

20. Zhou J, Gugger ET, Tanaka T et al. (1999) Soybean phytochemicals inhibit the growth of transplantable human prostate carcinoma and tumor angiogenesis in mice. J Nutr 129, 1628 1635. 
21. Kumi-Diaka J, Butler A. (2000) Caspase-3 protease activation during the process of genistein-induced apoptosis in TM4 testicular cells. Biol Cell 92, 115-124.

22. Shi Y. (2002) Mechanisms of caspase activation and inhibition during apoptosis. Mol Cell 9 , 459-470.

23. Takahashi Y, Lavigne JA, Hursting SD et al. (2004) Using DNA microarray analyses to elucidate the effects of genistein in androgen-responsive prostate cancer cells: Identification of novel targets. Mol Carcinog 41, 108-119.

24. Suzuki K, Koike H, Matsui H et al. (2002) Genistein, a soy isoflavone, induces glutathione peroxidase in the human prostate cancer cell lines LNCaP and PC-3. Int J Cancer 99, 846852.

25. De Boer L, Oakes V, Beamish H et al. (2008) Cyclin A/cdk2 coordinates centrosomal and nuclear mitotic events. Oncogene 27, 4261-4268.

26. Husdal A, Bukholm G, Bukholm IRK. (2006). The prognostic value and overexpression of cyclin $\mathrm{A}$ is correlated with gene amplification of both cyclin $\mathrm{A}$ and cyclin $\mathrm{E}$ in breast cancer patient. Cell Oncol 28, 107-116.

27. Soni DV, Sramkoski RM, Lam M et al. (2008) Cyclin B1 is rate limiting but not essential for mitotic entry and progression in mammalian somatic cells. Cell Cycle 7, 1285-300.

28. Shore GC, Viallet J. (2005) Modulating the Bcl-2 family of apoptosis suppressors for potential therapeutic benefit in cancer. Hematology 1, 226-230.

29. Kumi-Diaka J, Hassanhi KM, Merchant K et al. (2006) Influence of Genistein Isoflavone on Matrix Metalloproteinase-2 Expression in Prostate Cancer Cells. J Med Food 9, 491-97.

30. Guo Y, Wang S, Hoot DR et al. (2007) Suppression of VEGF-mediated autocrine and paracrine interactions between prostate cancer cells and vascular endothelial cells by soy isoflavones. J Nutr Biochem 18, 408-417.

31. Ladd AN, Nguyen NH, Malhotra K et al. (2004) CELF6, a Member of the CELF Family of RNA-binding Proteins, Regulates Muscle-specific Splicing Enhancer-dependent Alternative Splicing. J Biol Chem 279, 17756-17764.

32. Paillard L, Omilli F, Legagneux V et al. (1998) EDEN and EDEN-BP, a cis element and an associated factor that mediate sequence-specific mRNA deadenylation in Xenopus embryos. EMBO J 17, 278-287.

33. Tomasini R, Samir AA, Carrier A et al. (2003) TP53INP1s and Homeodomain-interacting Protein Kinase-2 (HIPK2) Are Partners in Regulating p53 Activity. J Biol Chem 278, 3772237729.

34. Takei Y, Ishikawa S, Tokino T et al. (1998) Isolation of a novel TP53 target gene from a colon cancer cell line carrying a highly regulated wild-type TP53 expression system. Genes Chromosomes Cancer 23, 1-9. 